\title{
A new relaying scheme for cheap wireless relay nodes
}

\author{
Ramin Khalili \\ Université Pierre et Marie Curie \\ LIP6-CNRS, Paris, France \\ ramin.khalili@lip6.fr
}

\author{
Kavé Salamatian \\ Université Pierre et Marie Curie \\ LIP6-CNRS, Paris, France \\ kave.salamatian@lip6.fr
}

\begin{abstract}
Wireless networks consist of senders, receivers, and intermediate nodes that collaborating (more or less) to establish the communication paths. Most of the researches in the domain of wireless network have focused on routing based approaches. In such an approach wireless network is reduced to a dynamic graph, and a minimum cost routing mechanism is applied. These approaches have led to several routing mechanisms as OLSR and AODV. However, the fundamental nature of wireless network is the broadcast. In the wireless network all the tuned receivers potentially receive every transmission. This basic property is not well captured by graph-based approaches where packets follow a single path from sender to receiver.

In this paper we will propose a relaying scheme for wireless multi-hop networks. It is based on collaboration of intermediate relays at network layer to forward useful side information in place of dumbly forwarding packets. In our scheme we assume that the nodes are not able to benefit from any interference cancellation mechanism. The channels from sender to relay nodes and from sender to receiver are logically separated through a temporal scheduling. This model is realistic for many practical scenarios in the context of wireless networks.

We will show in this paper the information theoretic bounds and show that they are achievable using practical codes. The proposed coding scheme is simulated in realistic scenarios. The obtained results show a remarkable improvement in throughput, relay load and reliability compared to network using classical routing approach.
\end{abstract}

\section{Introduction}

Formally, a relay network is a network with one sender, one receiver and a number of intermediate nodes. The intermediate nodes participate in the communication by relaying the packets from the sender to the receiver. The capacity region of relay networks remains unknown in the general case. Until now, most of the researches on the relay channel have focused on the case of error channel with interferences between sender and relay transmission. The capacity is only known for the simple case of relay network, composed of a unique intermediate node, and where the relay channel is physically degraded $[6,22]$. For this scenario, it has been shown that interference cancellation greatly improves the achievable rate. However, in many wireless network architectures, we are not able to use interference cancellation. This means that classical approaches toward relay channels, as proposed in [22,9], are not applicable. Therefore the new bounds for this particular scenario have to be defined.

This is the case in practical Ad hoc networks where we have to use existing physical layer card that are not yet able to apply interference cancellation. In these cases, each node has a single wireless interface card that is used for transmission and reception at the same time. In a very practical setting, the WIFI interface with a CSMA/CA based MAC layer is used. This setting will be analyzed in this work. This means that we assume that we cannot make any changes at physical layers to take benefits from interference cancellation. These nodes are called "cheap node" compared to nodes used in Wireless Mesh Router [2] which have several physical interfaces or the nodes that are able to implement interference cancellation.

In this scenario, interferences (collisions) between concurrent senders are mitigated through scheduling the transmission of each competitor. The CSMA/CA mechanisms are aimed toward the goal of providing distributed scheduling mechanisms in a wireless network. However, these approaches might not be able to suppress completely collisions (because of far sender). If collision occurs, the packet is supposed to be lost and have to be retransmitted (or recovered). Packets might also be erased by link layer error-detection mechanisms such as Forward Errorcorrecting Codes (FEC). These errors are induced by the physical environment (noise, multi-path, etc.) as well as other senders co-channel interferences. The channel therefore appears as an erasure channel. Each receiver might receive packets sent by any sender with a reception proba- 
bility. This reception probability is a function of the topological setting (the distance between senders and receivers), emission power, resulting level of interference, and coding characteristics.

This important property is sometime overlooked in the information theoretical literature and it results in a lot of simplification in analysis. This enables the derivation of simple closed form bound [21, 12]. No simple formulation are available on error channels with similar setting. Moreover, MDS codes [23] are known to be sphere packing codes for the erasure channel. There exists practical MDS codes achieving optimally the capacity of erasure channels.

Most of the researches in the domain of wireless network have focused on routing based approaches. In such an approach the wireless network is reduced as a dynamic graph on which a minimum cost routing mechanism is applied. Several routing mechanisms such as OLSR (Optimized Link State Routing Protocol) [5] or AODV (Ad hoc On Demand Distance Vector) [19] are the examples of routing-based approaches. As said before, the fundamental nature of wireless network is broadcast. All the receivers tuned to the sending channel potentially receive every transmission. This basic property is not well captured by graphbased approaches where, packets follow a single path from sender to receiver. Using a multi-path approach is very attractive; however, Optimal MultiPath routing shown to be NP-Complete. On the other extreme, packet flooding approaches that also use all the available capacity in the wireless network waste a lot of energy.

Recently, network coding $[1,16,18]$ has been proposed as a new paradigm to look at the issue of network capacity. The core notion of network coding is to allow and encourage mixing of data at intermediate network nodes. Network coding defines a new type of collaboration schemes. This collaboration scheme consists of mixing the received information through a coding scheme defined for each node and to forward the encoded version. Classical forwarding scheme sends an exact copy of each received packet over another link is a very specific case of network coding. In this case coding reduces to copying a packet. Initially network coding had been developed in the context of communication graphs where no losses or errors occur for each transmission. Extending classical network coding to wireless network where losses can occur at each transmission is not straightforward. Moreover most of network coding mechanisms are based on random coding scheme that might be difficult to use in a realistic situations. Some new efforts have been made in the past couple of year to provide practical network coding schemes. For example [3] proposed a distributed scheme for practical network coding which allows the intermediate nodes to merge received packets. The provided mechanism remains random as the network coding acts by randomly adding arriving packets and hoping with high probability. The received packets at the receivers create a sufficient number of linearly independent packets to be able to retrieve initial data. [3] further present some simulation results. The simulation has done in a graph of ISP backbones and it is shown that in practical the network coding could improve the performance with a reasonable transmission delay. However as said before graph-based approaches does not capture the broadcast nature of wireless networks and some modification must be apply in the context of network coding to be adapted to the wireless networks area.

In this paper we present a coding scheme which directly exploits the broadcast nature of wireless communication. The proposed coding scheme tries to reach the overall capacity of the network without flooding it with redundant packets. The approach is rooted in information theoretic notion. It is based on the collaboration of intermediate relays at network layer to forward useful side information in place of forwarding packets dumbly. The main idea is to diffuse the side information on the space and, benefit from the proposed coding scheme to make the collaboration between the nodes. This method does not benefit from any type of routing algorithm. That is the wireless channel which finds the best way to route the packet to the destination. Moreover, the proposed coding scheme can be readily implemented on an actual WIFI based wireless network. It does not need any change in the physical layer.

[13] presents the capacity region of the degraded single relay erasure relay channel when interferences are suppressed by using different physical channel. It is shown that this capacity region can be attained by a practical coding scheme. The main idea of the proposed coding scheme comes from the coding scheme proposed by Cover $\& a l$. in [22]. It suggests that the relay should only transmit useful side information for the decoding at the final receiver. This is completely different from classical approaches that make a copy of packets and retransmit them [9]. The capacity region and coding scheme was extended to the non-degraded single-relay erasure relay channel in [12].

In this paper, we will extent the approach to the case of realistic relay node with a single physical interface. Channel separation is done through temporal scheduling. CSMA/CA mechanism offers a distributed mechanism for such temporal scheduling. We use here the same coding scheme as proposed in [12]. We will present the capacity region for the case of cheap relay nodes that the nodes have a single physical interface and where, interferences are suppressed by scheduling the transmission of the sender and relay nodes. A similar problem is addressed in [15] which present an upper bound based on cut-set argument for information transfer rate over a general relay network. We show here that the coding scheme presented in [13] and [12] attains the cut-set bound presented in [15] in the special case 
of physically degraded channel, meaning that this is bound is in fact the capacity region.

We will show how the proposed scheme can be applied in a practical multiple relaying situation. The performance of the proposed scheme is compared with the widely referred AODV routing scheme through a simulation under NS-2.26 simulator. This comparison shows a remarkable improvement for the throughput of the network compared to classical routing based schemes especially in the case of large networks.

The paper is organized as follow. Section II gives theoretical results on the capacity of single relay case and extends theoretical results to multi-relay case. Section III introduces the coding scheme for the erasure relay network that attains the theoretical bounds for the case of multirelay. The practical implementation and the simulation results are presented in section IV.

\section{Information Theoretical bounds}

This section will present theoretical bounds for the capacity of a specific type of relay channel. We assume that all nodes operate over a unique and common physical channel and they are not able to apply the interference cancellation at physical layer, i.e., each possible transmission can potentially be an interference for other transmissions and lead to collision. Moreover, we assume that the nodes are not able to send and receive simultaneously. Reception from multiple nodes at the same time is also impossible as it leads to collisions. A scheduling mechanism must be applied in such a network to separate the interfering communications. Each node in the network might be in two states: sending (S) and receiving ( $\mathrm{R})$. The scheduling algorithm defines different transmission mode for the relay network by assigning to each node a state ( $\mathrm{R}$ or $\mathrm{S})$. Collisions occur in a mode if two or more senders are assigned to the $\mathrm{R}$ state by the scheduler. If only one node is assigned to the $\mathrm{R}$ state and all other nodes are assigned to $\mathrm{S}$ state, the message sent by this unique sender might be received by all other nodes with a probability related to Packet Error Rate that depends on distance and transmission power. For the sake of simplicity, we first begin with the simple case of a single relay shown in Fig. 1 and we will extend the analysis to multiple relay situations shown in Fig. 2.

\subsection{Single-relay case}

In this scenario, the scheduler defines four different modes. In mode $m_{1}$ node $S$ send messages and nodes $D$ and $R$ receive the message with a specified probability that depends on distance and transmission power. In this mode the relay channel appears as an erasure broadcast channel $\left(X_{m_{1}}^{0} ; Y_{m_{1}}^{0}, Y_{m_{1}}^{1}\right)$ as defined in [21]. In the second mode

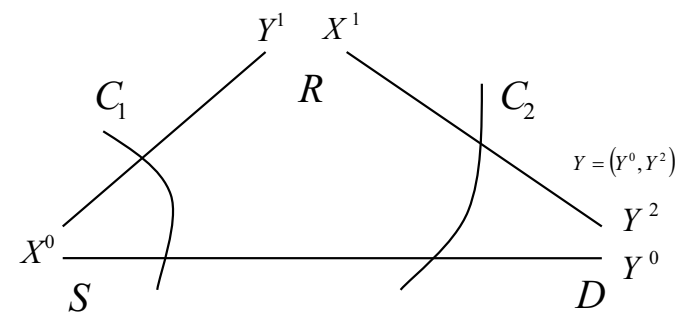

Figure 1. The single-relay scenario

$m_{2}$, the relay node acts as the sender and the node $D$ is the receiver. In this case the channel appears as a point to point erasure channel $\left(X_{m_{2}}^{1} ; Y_{m_{2}}^{2}\right)$. In mode $m_{3}$, the relay and sender are in sending state and collision occurs. In this mode the receiver do not receive any information. In the last mode $m_{4}$, all the nodes are in receiving state and no information is transferred through the packets. Let suppose that the $t_{i}$ is the proportion of time that the wireless network is in state $m_{i}$. Clearly $\sum_{m=1}^{M} t_{i}=1$, meaning that the scheduling mechanism do a time-sharing between the different possible modes.

The previous description might be generalized in a straightforward way to a network with more that one intermediate nodes. In this setting any possible assignment of state value to each node define a transmission mode $m_{i}$ that is active a proportion $t i$ of time. We will assume for the sake of tractable theoretical analysis that the scheduling is deterministic, meaning that the scheduling is defined in advance independently of transmission results. This hypothesis is clearly not valid if CSMA/CA mechanism is used for defining the scheduling in a distributed way. However, the evaluation of the proposed relaying mechanism that will be presented in section IV will use the CSMA/CA mechanism. That section will show that the hypothesis of deterministic scheduling is not critical.

In [15] a cut-set bound is derived for the achievable rate over a general multi-mode relay channels is derived. We use this bound and specialize it to the specific case of erasure relay channel.

Theorem 1 (Cut-set Bound [15]) Consider a general network with $N$ nodes and a finite number of states, $M$. Now suppose that state of network is a deterministic function for every network use $k$ as $m_{k}$ and is fixed and is known to all nodes then

$$
\sum_{i \in S, j \in S^{c}} R^{i j} \leq \sum_{m=1}^{M} t_{m} I\left(X_{(m)}^{S}, Y_{(m)}^{S^{c}} \mid X_{(m)}^{S^{c}}\right)
$$


for all $S \subset\{1,2, \ldots, N\}$, where the set of all nodes are partitioned into two disjoint set $S$ and $S^{c}$ by cut-set. The portion of time that network operate in mode $m$ is defined as $t_{m}=\lim _{k \rightarrow \infty} n_{m}(k) / k$. For any state $m, n_{m}(k)$ is defined as the number of state which is equal to $m$ in the first $k$ network uses.

From theorem 1, an upper bound for the information transfer rate $R$ from the source node $S$ to the destination node $D$ in Fig. 1 can be derived as :

$$
\begin{aligned}
R \leq & \sup _{0 \leq t_{1} \leq 1} \min \left\{t_{1} \cdot I\left(X_{m_{1}}^{0} ; Y_{m_{1}}^{0}, Y_{m_{1}}^{1}\right)+t_{2} \cdot I\right. \\
& \left(X_{m_{2}}^{0}, Y_{m_{2}}^{2} \mid X_{m_{2}}^{1}\right), t_{1} \cdot I\left(X_{m_{1}}^{0} ; Y_{m_{1}}^{0}\right)+t_{2} . \\
& \left.I\left(X_{m_{2}}^{0}, X_{m_{2}}^{1} ; Y_{m_{2}}^{2}\right)\right\}
\end{aligned}
$$

Over the two choices of subsets $C_{1}$ and $C_{2}$ shown in figure 1 , where the parameter $t_{1}$ represent the asymptotic proportion of time that the network operate in mode $m_{1}$.

This bound might be simplified thanks to the Shearer theorem [4] for erasure relay channel.

Theorem 2 (Shearer Theorem [4]) Let $X^{n}$ be a collection of $n$ random variables and $Z^{n}$ be a collection of $n$ boolean random variable, such that for each $i, 1 \leq i \leq n$, $E\left\{Z_{i}\right\}=1-\tilde{C}$. If $X^{n}\left(Z^{n}\right)$ is a sub-collection containing the $i^{t} h$ random variable $X_{i}$ if $Z_{i}=1$. Then $E\left\{H\left(X^{n}\left(Z^{n}\right)\right)\right\} \geq \tilde{C} H\left(X^{n}\right)$

$$
E\left\{H\left(X^{n}\left(Z^{n}\right)\right)\right\} \geq \tilde{C} H\left(X^{n}\right)
$$

This lead to the following upper bound for a single relay erasure relay channel, under virtually degraded hypothesis. This hypothesis is equivalent to saying that the sender $S$ to relay $R$ channel is better than the sender $S$ to receiver $D$ channel. Under this setting :

Theorem 3 The cut-set upper bound for the specific case of physically degraded erasure relay channel described in Figure 1 is given by:

$$
R \leq \sup _{0 \leq t_{1} \leq 1}\left\{\min \left\{t_{1}\left(1-p_{1}\right), t_{2}\left(1-p_{2}\right)+t_{1}(1-p)\right\}\right\}
$$

where $p$ is the loss probability of the sender $S$ to receiver $D$ channel, $p_{1}$ is the loss probability of the sender $S$ to relay $R$ channel and $p_{2}$ is the loss probability of the relay $R$ to receiver D channel

This bound might be extended easily to a stationary ergodic setting with fading. Moreover, it can be readily extended to the non degraded situation through the approach presented in [12]. However, for simpler notation we will describe here only the virtually degraded case.

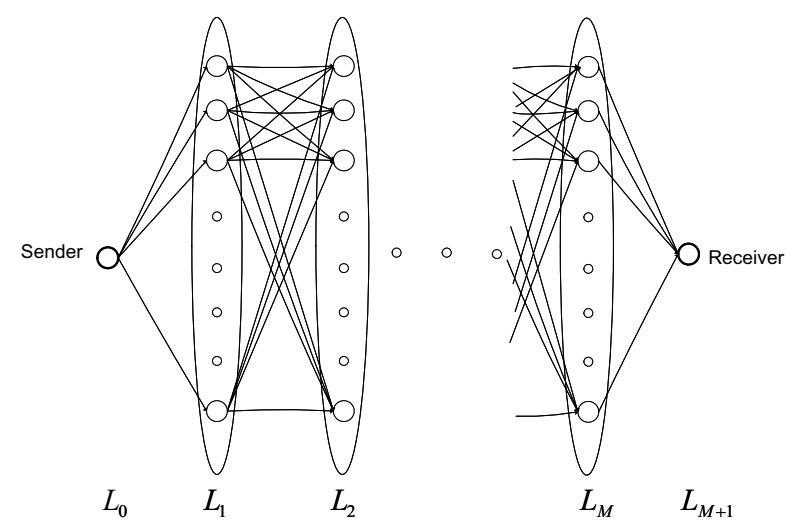

Figure 2. The Multi-relay scenario

\subsection{Multi-relay case}

This relationship might be extended in a straightforward way to the more general case of multi-relay network as depicted in Fig. 2. Here we borrow some of the notations presented in [8] for describing this multi-relay setting. Lets consider a set of $N$ node $\mathcal{N}=\{1, \ldots, N\}$ communicating over a general erasure channel characterized by a loss matrix $\mathbb{P}=\left(p_{i, j}\right)_{N \times N}$, where $p_{i, j}$ is the loss probability of a transmission between node $i$ and $j$. Now let's suppose that there is a single communication taking place between a single sender-destination pair $(s, d)$.

Let's suppose that nodes in $\mathcal{N}$ are partitioned into $M+1$ disjoint level sets $\left(L_{0}, L_{1}, \ldots, L_{M}, L_{M+1}\right)$ where $L_{0}=\{s\}$ is the sender node and $L_{M+1}=\{d\}$ is the destination node. We assume that the nodes in $L_{m}$ only receive information from the nodes in previous levels $L_{m-k}$ for $k \in[1, m]$, while they only send information to the nodes in the next levels $L_{m+k}$ for $k \in[1, M+1-m]$. Let $n_{m}$ be the number of nodes in level $L_{m}$ by defining $n_{0}=n_{M+1}=1$. (see Fig. 2). In such a scenario, the cut-set bound presented in theorem 1 can be simplified thanks to the Shearer theorem as bellow.

Theorem 4 (Capacity bound for erasure multi-relay case) The capacity region of a degraded erasure multi relay channel where , $p_{m j, s q}$ is the loss probability from node $j$ in $L_{m}$ to node $q$ in $L_{s}$ is bounded by:

$$
R<\max _{t}\left[\min \left\{R^{1}, R^{2}, \ldots, R^{M}\right\}\right]
$$

where the maximization occurs over all possible mode proportion $\left\{t_{i}\right\}_{i=1}^{M}$. Moreover $R^{u}, u=1, \ldots, M$ is defined as

$$
R^{u}=\leq \sum_{m=0}^{u-1} \sum_{j=1}^{n_{m}} t_{m j} \cdot\left(1-\prod_{q=1}^{n_{u}} p_{m j, u q}\right)
$$


$t_{m j}$ is the asymptotic proportional of time that the node $m$ in level $L_{j}$ acts as a sender.

Because of the lack of the place the proof is not presented here and can be find in [14].This theorem defines the partitioning and the scheduling as abstract notions. Clearly, the bound depends on the chosen partitioning as well as the scheduling of nodes. This means that the capacity region might be enlarged by optimizing the scheduling and the partitioning. This subject is out of the scope of this paper as we wish here to present a practical setting that might even be applicable to CSMA/CA based scheduling in realistic Ad-hoc networks. Therefore we assume that the partitioning results from the scheduling mechanism as well as the topological properties of the wireless network. In next section, we will present a coding scheme achieving the previous bound.

\section{Achievability and Coding Method}

In this section, we present a coding scheme that attains the capacity bound presented in the previous section. The coding scheme is the same as what presented in [13]. We first describe the single relay case and then extend the approach to the multi-relay case.

\subsection{Single-relay case}

As explained before, because of error detection mechanisms at the link layer, the transmission channel of wireless networks as seen from higher layers can be modeled by an erasure channel acting over the large input alphabet formed by IP packets. Specific codes have been designed to deal with erasures in place of errors. It is worth mentioning that the class of Maximal Distance Separable (MDS) [23] code leads to sphere packing codes for erasure channels. Let's suppose a systematic $(n, k)$ linear code described by an encoding and decoding matrix. Let's suppose that a vector $w \in \mathcal{X}^{k}$ of information is encoded to a vector $v \in \mathcal{X}^{n}$ by $v=w\left[I_{k \times k} \mid A_{k \times(n-k)}\right]$. The vector $v$ contains as its first $k$ symbols the vector $w$ as well as $(n-k)$ redundant symbols. If all sub matrices of $\left[I_{k \times k} \mid A_{k \times(n-k)}\right]$ are invertible the resulting code will be MDS. Reed-Solomon codes are well known and widely applied members of these classes of codes. A $(n, k)$ MDS code with rate $R=\frac{k}{n}$ has the property that any combination of $k$ encoded symbols out of the $n$ encoded symbols enables to retrieve the initial $k$ unencoded symbols. In other word MDS codes can correct at most $n-k$ erasures in a block of $n$ symbols.

An MDS code can achieve the capacity of a stationary and ergodic erasure channel asymptotically with a larger block size $n$ if its rate $R$ is less than the capacity of the channel. Moreover the encoding and decoding complexity of MDS codes is $\mathcal{O}(n \log n)$, which makes them interesting in practical settings. More recently, the class of Tornado codes has defined almost-MDS codes that have a linear encoding and decoding complexity. However MDS codes have a delay cost: the decoding cannot begin without the reception of at least $k$ symbols from a block, i.e. a delay bounded by the time needed to receiving $n$ packets. This might generate some trouble for applications with stringent delay constraints and a trade-off between code performance and delay should be made. Convenient properties of MDS codes have made them very attractive for network communications especially in the context of broadcast channels $[10,21]$. We will present here a coding scheme based on MDS code which achieves the bound presented in the theoretical section.

Before going further into the description of the coding scheme, we have to describe more carefully the action of an MDS code. Let's suppose that a $(n, k)$ MDS codes is defined over a symbol set $\mathcal{X}$. For an erasure channel the output set of the channel will be $\mathcal{Y}=\mathcal{X} \cup\{\mathrm{e}\}$ where e is the erasure symbol. The MDS code divide the space $\mathcal{Y}^{n}$ with $(|\mathcal{X}|+1)^{n}$ points in $|\mathcal{X}|^{n}$ separated cosets containing each $\sum_{l=0}^{k}\left(\begin{array}{c}n \\ n-l\end{array}\right)$ points. The cosets need therefore an index with $n \log |\mathcal{X}|$ bits to be addressed. Each received packet in a block can be seen as a part of the index needed for finding the correct coset and lead to a correct decoding.

Consider the erasure relay network of figure 1 where the sender $S$ has $k$ packet to transmit. Now let's suppose we have designed an $(k+m+l, k)$ MDS code with an encoding matrix $\left[I_{k \times k}\left|A_{k \times m}\right| B_{k \times l}\right]$. At the sender we encode these packet with the $(n=k+m, k)$ MDS code with encoding matrix $\left[I_{k \times k} \mid A_{k \times m}\right]$ leading to $m$ redundant packets and a rate $R^{s}=\frac{k}{n}$. These redundant packets will help the receiver and the relay to retrieve the erased packets over the channel. Under the condition that $R^{s}<\left(1-p_{1}\right)$ a MDS code will asymptotically, with large $k$ and $m=k\left(\frac{1}{R^{s}}-1\right)$, ensure perfect communication between the sender and relay, as the MDS codes achieve the capacity of the erasure channel.

At the receiver side we will receive asymptotically, with large $n$, around $n(1-p)$ packets from the sender and $l(1-$ $\left.p_{2}\right)$ packets from the relay. The receiver has to decode the MDS code with the generator matrix $\left[I_{k \times k}\left|A_{k \times m}\right| B_{k \times l}\right]$. The $k$ message packets can be recovered if we receive from the sender and the relay at least $k=n R^{s}$ packets. Using this coding scheme, the relay only send useful index information to the receiver. The fact that $\left[I_{k \times k}\left|A_{k \times m}\right| B_{k \times l}\right]$ is the generator of an MDS code guarantees that every packet received from the relay will reduce the ambiguity about the initial message.

Asymptotically, with large $l$ (or equivalently large $n$ ), receiver will receive together, $n(1-p)+l\left(1-p_{2}\right)$ packets from sender and relay. The MDS code with encoding matrix 
$\left[I_{k \times k} \mid A_{k \times m \mid B_{k \times l}}\right]$ might be decode if $n(1-p)+l(1-$ $\left.p_{2}\right)>k$, i.e., $R^{s}<(1-p)+\frac{l}{n}\left(1-p_{2}\right)$. Moreover, with the scheduling mechanism the sender only transmits a proportion $t_{1}$ of time and the relay a proportion $t_{2}$ which leads to the transmission rate

$R=t_{1} \cdot R^{s}=\sup _{0 \leq t_{1} \leq 1}\left\{\min \left\{t_{1}\left(1-p_{1}\right), t_{2}\left(1-p_{2}\right)+t_{1}(1-p)\right\}\right\}$.

This shows that the capacity region bound defined for the degraded situation is achievable by the proposed scheme. In practice the number of the codewords sent by the relay $(l)$ can be chosen as $l=n \frac{t_{2}}{t_{1}}$ to guaranty the maximum possible rate. This coding scheme can be extended in a straightforward way to the general case of multi-relay.

\subsection{Multi-relay case}

The presented scheme for the single relay case can be extended in a straightforward way to the multi-relay case. We just do the same think for all relay nodes in the wireless network. Every relay $i$ will have its proper code $B_{k \times l_{i}}^{i}$ known by all other relays. This code acts similarly to an access code in CDMA. Similarly to the single relay case, the main constraint is that the overall generator matrix

$$
C=\left[I_{k \times k}\left|B_{k \times(n-k)}^{0}\right| B_{k \times l_{1}}^{1}|\ldots| B_{k \times l_{N}}^{N}\right]
$$

is an MDS code generator, i.e., every squared sub matrix of it being invertible. Note that we represent here the encoding matrix of the sender as $B_{k \times(n-k)}^{0}$. Now at each relay in the network we receive packets coming from the source as well as from the previous relay layers. If these received packets reach a sufficient number (more exactly $k$ packets), they are decoded using the encoding matrix of the source of these packets. The decoded block is thereafter re-encoded using the particular code of the relay $B_{k \times l_{i}}^{i}$ leading to $l_{i}$ side information packets that are sent over the channel. This process is continued until the final destination that will be able to decode the sent block of data if it receives $k$ packets. The only point in the mechanism is to verify the basic hypothesis of the partitioning which was that no packet from layer numbers larger than $k$ should be processed in layer $k$. This can be enforced by adding to packets a header containing a block number. A block number that has been treated, decoded, and re-encoded by a relay is not anymore treated. Moreover to reduce the number of useless redundant packets forwarded we might use a TTL (Time To Live) mechanism. It decrease a counter set at the sender after crossing each relay assure that the packet will not be forwarded anymore when the counter goes down to 0 . This mechanism can be shown to reach the capacity bound presented in theorem 4 . This shows in the fact that the capacity bound given in theorem 4 is the capacity region of the multi-relay erasure channel.
In next section, we will validate the proposed relaying scheme in a practical scenario through a simulation under NS-2.26 environment. The simulation will also enable comparison of the approach with more traditional routing based approach following the AODV protocol.

\section{Validation by simulation}

\subsection{Simulation settings}

In this section, we will describe the simulations done under the NS-2.26 environment. We will also demonstrate that the proposed scheme can be easily implemented in real WiFi based wireless networks since it does not need any change to physical layer architecture.

We developed in NS a new routing agent implementing the proposed coding scheme where each relay uses its specified codes. We added also to IP packets a new header with a field containing the block number. This header is used to recover packets belonging to the same block in order to decode it. We added also a TTL counter in order to avoid an exponential flooding of the network. In order to evaluate the amount of supplementary packet sent by the mechanism we monitor in all simulations the relay load which is defined as the mean number of needed transmission for sending a data packet from source to destination. We do not make any change to the IEEE 802.11B MAC layer implementation, as the proposed scheme does not require such changes. The scheduling and partitioning therefore results from the distributed action of wireless MAC layer. However, all relays send in broadcast mode meaning that WiFi retransmission mechanism that retransmits the packet lost due to erroneous reception is bypassed in the proposed scheme. In order to evaluate the impact of retransmission in the comparison between the proposed scheme and AODV based scheme we have also measure the reliability of the proposed scheme. The reliability is defined as the probability that a packet sent by the sender is never received by receiver and might therefore need a retransmission from sender.

The CSMA/CA scheduling mechanism does not suppress completely collisions in the network and they occur frequently. However, MAC layer CSMA/CA mechanisms mitigate the collisions and if a collision occurs, the two colliding packets are supposed to be lost but as said before the packets are not retransmitted. Therefore, the total packet error rate is the sum of the packets lost because of error caused by channel noise and lost packet due to collision.

The Packet Error Rate due to channel noise is simulated using a model based indirectly on distance via Signal to Noise Ratio at receiving node. The larger the distance is the smaller the SNR and the larger the Packet Error Rate (PER) will be. The relation between SNR and PER follows a formula previously derived in [11]. NS also implement 
a receiver sensitivity mechanism that prohibits a receiver of receiving packets if the SNR is below a threshold. This threshold set the range of a sender to around 100 meters for a transmitting power of $P_{\max }$. We have also used a Tworay ground reflection model for generating an attenuation exponent of 2,5.

Each relay node implements the proposed coding scheme. It listens to the channel and decides to allocate a buffer in memory for each new coding block observed. Arriving packets are stored in these buffers until enough packets are received to decode the corresponding block. The block is decoded and re-encoded using the relay specific code. These encoded packets are thereafter forwarded to the MAC layer and sent over the medium and the buffer is freed. The buffer is also freed if after timer expiration we have not received enough packets for the decoding. If a relay decodes a block and finds out that he is the destination, it is forwarded to upper layers. The traffic is simulated by using a CBR sources with rate is $2 \mathrm{Mbps}$ and the packet size is set to 1500 bytes. The MDS block size was set to $N=10$. The sender code rate is calculated to optimize the relay load and transmission rate following the method proposed in [12].

We run the simulation over several random topology scenarios. However we present here only two typical scenarios : a dense scenario with 30 nodes in an area of $600 \times 600 \mathrm{~m}^{2}$ (see figure $3(\mathrm{a})$ ) and a more spread scenario with 30 nodes in an area of $1000 \times 1000 \mathrm{~m}^{2}$, figure $3(\mathrm{~b})$. In each case one couple of sender and receiver are chosen randomly.

We compared the proposed method with an AODV routing scheme [19]. AODV is largely referred in the literature as a routing protocol that is well suited for WiFi type Adhoc networks. The simulations are done using the implementation of AODV in NS-2.

\subsection{Simulation results}

In this section we present and analysis the results obtained by simulation. Each simulation round $i$ consists of setting the power level of each sender at a value $P=$ $\beta_{i} P_{\max }$ and running the simulation for the proposed scheme as well as a AODV based approach. The results are stored on a trace file that is used to derive three main parameters of interest.

- The throughput in term of bits/sec calculated over the transmission time between sender and receiver.

- The relaying load defined as the number of packets sent in the environment for each packet data received by the receiver application layer.

- The mean delay calculated as the mean delay observed by received packets. This delay calculate as the differ-

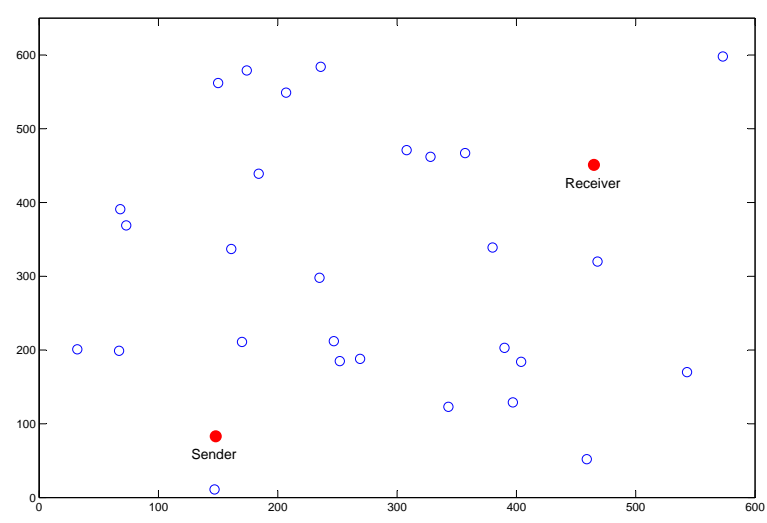

(a) Scenario 1: 30 nodes located randomly in an area $600 \times 600 \mathrm{~m}^{2}$.

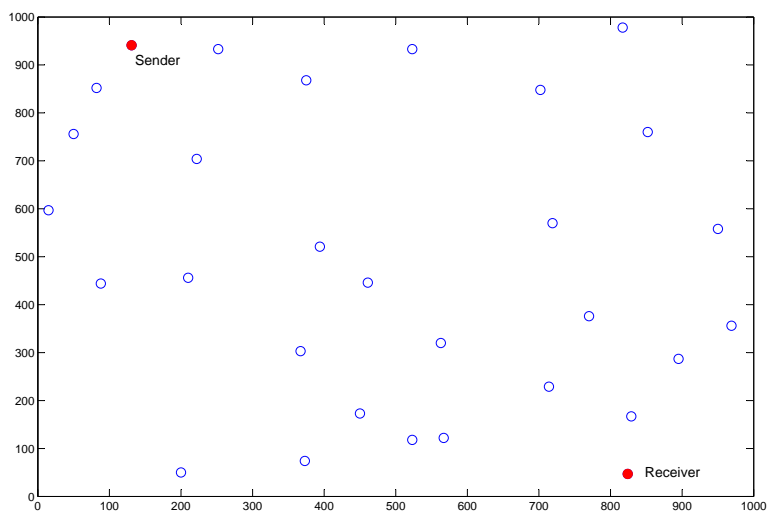

(b) Scenario 2: 30 nodes located randomly in an area $1000 \times$ $1000 \mathrm{~m}^{2}$.

\section{Figure 3. The two different scenarios used in the simulations}

ence between the time they are sent by the application at the source to the time they are received by the application layer at the receiver.

The comparison of the different scheme is bases on these three criteria. Each simulation round is done for AODV as well as for our proposed relaying scheme. The duration of each simulation round is $10 \mathrm{~ms}$. For each round we vary $\beta_{i}$ by steps of 0.1 varying between 0 and 1 .

\subsection{Scenario 1}

We first compare the three performance metrics in the dense scenario. The attained throughput is shown in Fig. 4(a) for the proposed relaying scheme and for AODV. As observed, the proposed scheme outperforms largely AODV for all node power scenarios. The gain can goes as high 
as 500 times for low transmission power to a factor of 1.3 for large transmission power. This gain was expected as the proposed scheme uses all the available capacity for the transmission where AODV just use the available capacity in one path. It is noteworthy that the proposed relaying scheme can attain a rather good rate even in very low transmission level power situation which make it attractive for situation where power consumption is a concern (as sensor nets). For higher power levels, the rate is bounded in the proposed scheme by a large increase in collisions coming from the density of the environment. However, for AODV, the rate is bounded by a single hop where the two relaying nodes in the path are far from each other. Collision affects AODV but to a smaller extend than the proposed relaying scheme. This explain why the proposed relaying scheme attain a plateau for transmission power in the order of $0.2 P_{\max }$ while AODV is still slightly increasing its throughput. It also says that it is almost useless of using a power larger than $0.2 P_{\max }$ by using the proposed scheme where with AODV a larger power might be needed to attain similar throughput.

To have a better understanding of what is happening in the network lets compare the relay load as shown in Fig. 4(b). This figure gives insight into the behavior of both methods and validates somewhat the intuition developed when seeing Fig. 4(a). At low transmission power in AODV most of sent packets are retransmitted as the SNR is low and therefore the PER is high. However, for the proposed relaying scheme we do not have retransmissions as we are using the broadcast function of WiFi cards that bypass it. With increasing transmission power the relay loads converges and the relay load of AODV become smaller than the relay load of the proposed scheme. Ultimately for a transmitted power $P_{\max }$ the relay load of AODV is around $15 \%$ lower but with a loss of $30 \%$ in throughput compared to the proposed scheme. This shows the marginal benefit of the proposed scheme.

Another criterion of comparison is the transmission delay. This is shown in Fig. 5(a). It is expected that AODV should have a smaller transmission delay than the proposed relaying scheme. The rationale behind this intuition is that on one hand proposed relaying scheme requires that a whole block of 10 packets being received before being able to decode the block and forward the new packets. This induces a large decoding delay. On the other hand we show that the proposed scheme reach a larger throughput and moreover as it is one kind of controlled flooding, packets find themselves the shortest path from source to destination. This two contradictory effects shape the delay behavior. It can be seen in Fig. 5(a) that for low transmission powers the delay of AODV is much larger than the proposed scheme. This can be explained by the observation that when power is low the PER become large and packets stay a long time in queues

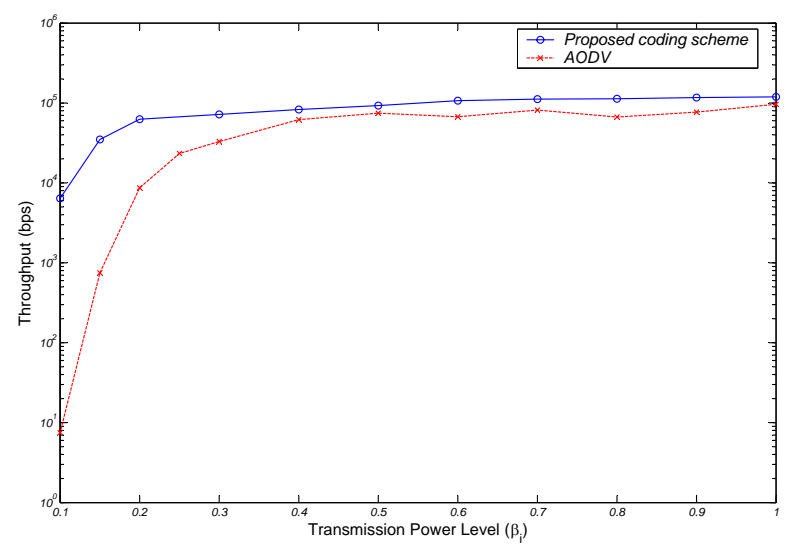

(a) Throughput performance

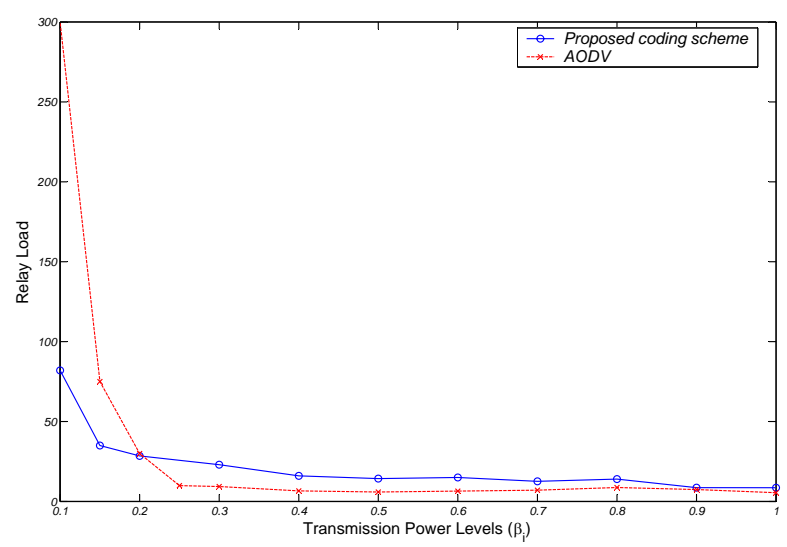

(b) Relay load

\section{Figure 4. The simulation results for topology No.1.}

waiting to be retransmitted. Because of this queues build up at nodes that are far from their next hop and congestion occurs which increase the delay. However, the congestion of intermediate node is bounded in the proposed relaying scheme by the fact that a block that has been treated and sent is not anymore stored. Meaning that queues do not build up and the crossing delay of a router is bounded by the time needed to receive a block and to decode it. The effects of this lack of congestion are visible in the average delay. For small transmission power where queue builds up because of retransmission and low throughput, the proposed scheme clearly outperform AODV. Nevertheless, when the transmission power increasing, AODV takes its revenge and show a smaller delay as expected. However, the comparison of the two methods shows that the proposed scheme seems to have good properties especially at low transmission power. Even if there is a slight increase in delay and 


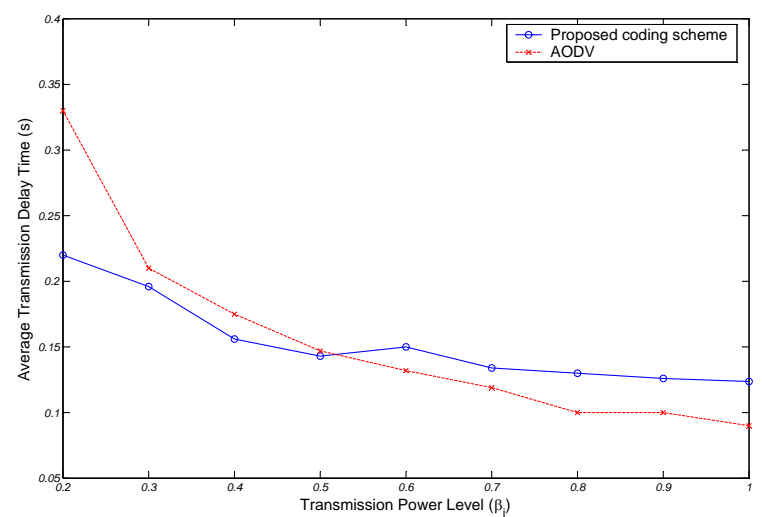

(a) The average transmission delay comparison

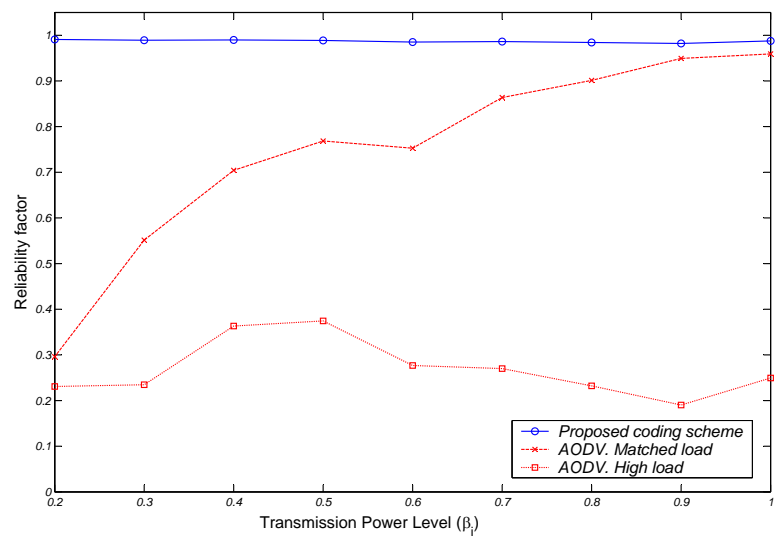

(b) The reliability factor comparison

\section{Figure 5. The simulation results for topology} No.1.

a slight relay load increase, the mechanism seems to have a good marginal benefit.

As a last analysis we compared the reliability factor of AODV and the proposed coding scheme in the figure 5(b). We define the reliability factor as the percentage of the packet transmitted by the sender and received by the receiver. When we are interesting to compare the performance of the protocols, we do not take into account the number of packets loss in the Interface Queue buffer. For AODV the simulation have been done for two different traffic loads, a high traffic load with rate $2 \mathrm{Mbps}$ and a lower traffic load which is approximately matched to the transmission rate of the network. When in the case of the proposed coding scheme the sender is able to estimate the maximum achievable rate of the network (as explained in the previous section) the sender transmits by its matched traffic load. We also consider that the MAC layer transmit in $11 \mathrm{Mbps}$. As it is shown in figure 5(b) AODV has a weak reliability factor especially in the case of high traffic load. There are two major reasons for this behavior of AODV : the first being that AODV uses a timer for each packet and if this timer expire the packet is dropped. The high transmission delay results in many packets drops in the intermediate (relay) nodes because of timer expiration. The second reason is that AODV does not retransmit a packet more than 5 times. Therefore, if a packet is dropped more than 6 times it is not retransmitted. This also explains the unreliability in the matched traffic load case. However, the proposed relaying scheme is almost perfect (the reliability rate is above $99.5 \%$ ).

\subsection{Scenario 2}

The second scenario is a sparse scenario with a smaller node density. It can be expected than the results should be similar in that case to the first scenario but with a smaller transmission power. Fig 6 validates this intuition; this figure appears to be a zooming of the initial part the Fig. 4. The same analysis as for scenario 1 can be applied to this scenario, with the notable difference that the situation is more favorable to the proposed relaying scheme.

\section{5 conclusion}

In this paper we presented a practical relaying scheme rooted on information theoretic. The main idea of the proposed scheme is to diffuse the information in the network and to gain from the natural diversity existing in the network, in place of pushing all data belonging to a sender to receiver connection to follow the same path. In this scheme every relay has a proper code similar to CDMA spreading code. This code is used by the relay to encode incoming information such that the side information sent by the relay to the network is not redundant. The coding scheme proposed has the nice property that the relaying decision is made only at relay node and the sender even does not need to be aware of the existence of a relaying node. The relays might even optimize the relay load the inject in the network if they have access to the packet loss probability matrix of the network $\mathbb{P}$. The proposed scheme can be easily implemented in actual WIFI based wireless network and it does not need any new physical layer architecture as needed for interference cancellation method proposed previously.

The presented approach is shown to outperform clearly AODV routing based approach at the cost of a slightly higher relay load for high transmission power situation. The approach presented here opens new approach for new relaying schemes for wireless and sensor network. Another direction we are going toward is the extension of the scheme to multi-sender, multi-receiver settings. This full extension would be topic of the future works. 


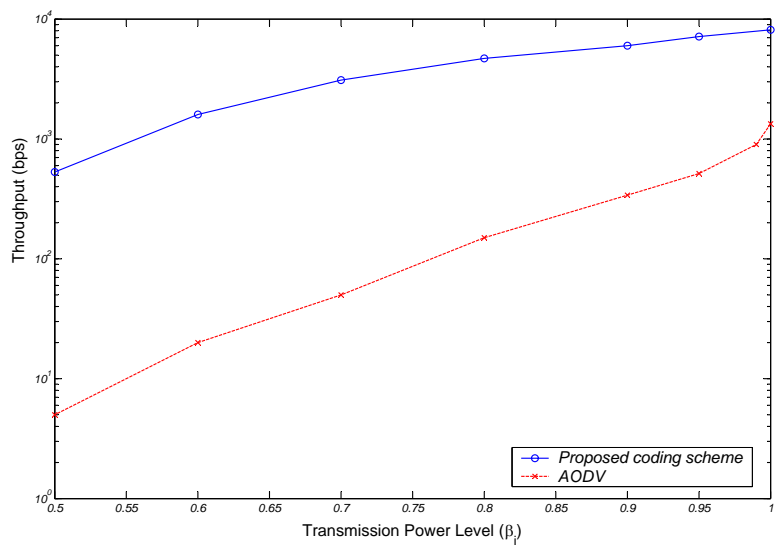

(a) Throughput performance

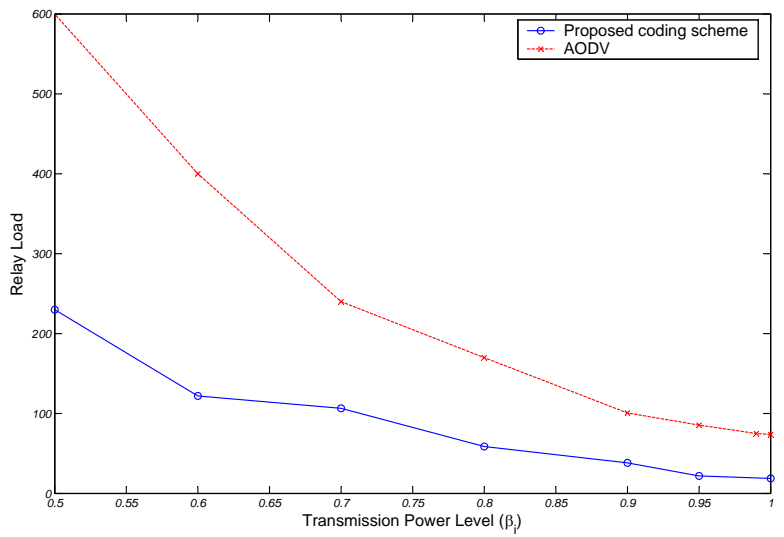

(b) The relay load comparision

Figure 6. The simulation results for the topology No.2.

\section{References}

[1] R. Ahlswede, N.Cai, S. R. Li, and R. W. Yrung. "network information flow". IEEE. Trans. Inform. Theory, IT-46:12041216, July 2000.

[2] D. Beyer. "fundamental characteristics and benefits of wireless routing ('mesh') networks". In Wireless Communications Association International, Technical Symposium, San Jose, California, Jan. 2002.

[3] P. A. Chou, Y. Wu, and K. Jain. "practical network coding,". Allerton, 2003.

[4] F. R. K. Chung, R. L. Graham, P. Frankl, and J. B. Shearer. "some intersection theorem for ordered set and graphs". Journ. of Comb. Theory, vol. 43(Series A):23-37, 1986.

[5] T. Clausen and P. Jacquet. "optimized link state routing protocol (olsr)". RFC 3626, Internet Engineering Task Force.

[6] T. M. Cover and J. A. Thomas. "Elements of Information Theory”. John Wiley Sons, New York, 1991.
[7] I. Csiszar and J. Korner. "Information Theory: Coding Theorems for Discrete Memoryless Systems". Academic press, 1981.

[8] P. Gupta and P. R. Kumar. "towards an information theory of large networks: An achievable rate region". IEEE. Trans. Inform. Theory, vol. IT-49:pp. 1877-1894, August 2003.

[9] P. Gupta and P. R. Kumar. "the capacity of wireless network". IEEE. Trans. Inform. Theory, vol. IT-46(no. 2):pp. 388-404, March 2000.

[10] J.Nonnenmacher, E.Biersack, and D.Towsley. "parity-based loss recovery for reliable multicast communication". ACM SIGCOMM '97, pages pages 289-300, Octobre 1977.

[11] R. Khalili and K. Salamatian. "a new analytic approach to evaluation of packet error rate in wireless networks". MSWIM2004, 2004.

[12] R. Khalili and K. Salamatian. "on the achievability of cutset bound for a class of erasure relay channels: The nondegraded case". ISITA, 2004.

[13] R. Khalili and K. Salamatian. "on the achievability of cut-set bound for a class of relay channels". IWWAN, 2004.

[14] R. Khalili and K. Salamatian. "on the achievability of the cut-set bound for a class of erasure relay channel: The multi relay case". Technical report, 2004.

[15] M. A. Khojastepour, A. Sabharwal, and B. Aazhang. "bounds on achievable rates for general multi-terminal networks with practical constraints ". Lecture Notes in Computer Science, LNCS, Vol.2634:pp. 146-161, April 2003.

[16] R. Koetter and M. Mdard. "an algebric approach to network coding”. IEEE/ACM Trans. Networking.

[17] J. Korner and K. Marton. "general broadcast channels with degraded messages sets". IEEE. Trans. Inform. Theory, IT23:60-64, 1977.

[18] S. R. Li, R. W. Yeung, and N. Cai. "linear network coding". IEEE. Trans. Inform. Theory, IT-49:371-381, February 2003.

[19] E. Perkins, E. Belding-Royer, and S. Das. "ad hoc on demand distance vector (aodv) routing". RFC 3561, IETF.

[20] K. Salamatian. ”Transmission Multimédia Fiable Sur Internet". PhD thesis, Université Paris SUD, 1999.

[21] S.Boucheron and M.R.Salamatian. "about priority encoding transmission". IEEE. Trans. Inform. Theory, on processing.

[22] T.M.Cover and A. E. Gamal. "capacity theorems for relay channels". IEEE. Trans. Inform. Theory, vol. IT-25:pp.572584, sep. 1979.

[23] F. Williams and N.Sloane. "Theory of Error-Correcting Codes". North-Holland, 1977. 